\title{
Zur Geschichte Ottheinrichs von Pfalz- Neuburg (1544)
}

von Dr. Adolf Hasenelever in Bonn.

1 .

A. v. Druffel hat in den Beilagen zur vierten Abteilung

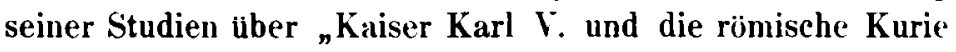
1544-1546"1) ein ,.undatiertes nicht uberschriebenes Aktenstuck“ zur pfälzischen Geschichte mitgeteilt. das „keinem gröBeren Zusammenhang angehört"; er bezeichnet es als „Memorial für eine geheime Unterredung des Pfalzgrafen Ottheinrich mit dem Kurfürsten Friedrich von der Pfalz", und glaubt seine Entstehung in die Zeit von Oktober bis Dezember $154 \bar{j}$ setzen zu sollen.

v. Druffel schließt diese Datierung hauptsaichlich aus einer, wie er annimmt. in der lrkunde enthaltenen Hindeutung auf die pfalz-neuburgische Landtagsicrhandlung im Januar 1546, sowie aus der Erwähnung eines Besuches des Kardinals von Augsburg. Otto von TruchseB-Waldburg, am Münchener Hof, der allerdings, wie wir aus den verschiedensten ${ }^{2}$ ) Quellen wissen, im Oktober 1545 stattfand. Eher könnte man an den April 1546 denken: damals

1) Abbandlungen der kgl. bayr. Akademie der W. Klasse III, Bd. XIX, Abt. 2, S. 495-500. Zu r. Druffels Anmerkung 2 auf S. 496 [S. 498] möchte ich bemerken, daß Friedrich der französischen Sprache durchaus mächtig war, wie ans State papers [King Henry VIII] Bd. XI S. 149 unzweifelhaft hervorgeht. Die angeführte stelle bei Leodius: de vita et rebus gestis Friderici II. etc. S. 85 schließt das anch nicht aus.

2) Monumenta Trideutina ed. Druffel No. 208 und Massarellis Tagebuch ed. Döllinger S. 111, 132, 133 (diese Quellen erwähnt Druffel auch S. 495 Anm. 1). Lenz: Bucerbriefwechsel Bd. III S. 361 Anm. 1 u. S. 377. - Riezler: die bayrische Politik im schmalkaldischen Kriege (Abhandlungen der Münchener Akademie Bd. XXI, Abt. 1) S. 156 ff. a. a. m. 
weilte der Kardinal von Augsburg ebenfalls im Auftrag des Kaisers am Yunchener Hof ${ }^{1}$ ); besonders aber wird die in dem Aktenstück erwähnte .hiesige Handlung." als etwas bereits der Vergangenheit Angehöriges betrachtet.

Doch aus dem ganzen Inhalt des Memorials geht unzweifelhaft herror, daB wir einen viel fruheren Zeitpunkt für die Abfassung des Aktenstuckes anzunehmen haben; $r$. Druffel selbst deutet das bereits an: ,ein anderer Anhaltspunkt würde sich ergeben, wenn man den Termin wubte, wo Ottheinrich nach Heidelberg ubersiedelte." ${ }^{2}$ ) Dieses Datum ist ziemlich genau bekannt: ${ }^{3}$ ) am 20. August 1544 wurde in Neuburg die Übergabeurkunde an die Stände des Landes vollzogen, daraufhin reiste Ottheinrich nach Baden zur Kur; ob er später für kurze Zeit in sein Furstentum zurìckgekehrt ist, wissen wir nicht, Ende Oktober ${ }^{4}$ ) war er jedoch nicht mehr in Neuburg "); schon am 26. Dezember 1544 kaufte er sich in Heidelberg zu bleibendem Wohnsitz ein Haus. Ind auch die ubrigen in der lirkunde erwähnten Ereignisse sprechen für den Herbst 1 j44 als Entstehungszeit des Aktenstuckes. Der Dank Ottheinrichs für die Bemühungen ${ }^{\text {) }}$ Kurfurst Friedrichs in "seinen Obliegen", besonders dab dieser jungst in der ,letzten und höchsten

1) Friedensburg: Nuntiaturberichte aus Deutschland I, Bd. VIII S. 602 und Bd. IX S. 9.

z) S. 495 Anm. 1.

3) Vergl. zum folgenden: R. Salzer: Beiträge zu einer Biographie Ottheinrichs (Heidelberg 1886) S. $78 \mathrm{f}$.

4) Auch die Notiz über den Besnch des Kardinals von Augsburg in München läßt sich hiermit in Einklang bringen: vom 16. Oktober ab weilte er als kaiserlicher Kommissar auf dem Wormser Reichstag. Winckelmann: Polit. Corr. v. Straßburg Bd. III S. 536. Da wir ron keiner Unterbrechung seiner dortigen Tätigkeit hören, ist sein Besuch vor Mitte Oktober anzusetzen.

3) Salzer S. 90.

๑) Persönlich scheint sich Friedrich von diesen Verhandlangen fern gehalten zn haben, obwohl er zar Entgegennahme der Huldigung damals in der Oberpfalz weilte. Mnffat: Geschichte der bayrischen und pfälzischen Kur. (Abhandl, d. bilyr. Ak. d. W. Bd. XI, Abt. 2) S. 292, und Leodius S. 260. 
Not" 1) ihm mit Rat und Beistand geholfen habe, kamn sich nur auf die Enterstiltzung Friedrichs bei den Vorverhandlungen zur Regelung seiner finanziellen Bedrängnisse beziehen. nicht auf die Teilnahme von kurpfälzischen Räten an den Landtagsberatungen ${ }^{2}$ ) im Januar 1546. Damals handelte es sich, soviel wir wissen. abgesehen von Widerstandsmabregeln gegen die Praktiken der Bayern, nur um die Frage. ob die neuburgische Landschaft dem schmalkaldisehen Bunde beitreten solle oder nicht. Besondere innere Schwierigkeiten scheinen nicht rorgelegen zu baben, umsoweniger als der heikelste Punkt, die Schuldentilyung. in geregelte Babnen geleitet war. ${ }^{3}$ )

Auch die Nachrichten iber Herzog Wilhelms von Bayern Verhältnis zu Ulrich von Wurttemberg lassen sich mit den guten Beziehungen dieser beiden Fursten im Herbst 1544 besser in Einklang bringen, als mit dem ziemlich gespannten diplomatischen Verkehr. wie er sich gegen Ende des Jahres 1545 zwischen den Höfen ron Stuttgart und Munchen wieder entwickelt hatte. ${ }^{4}$ ) Damals dachte auch niemand mehr

1) Denselben Aasdruck gebraucht Ottheinrich in einem Schreibeu an Herzog Johann v. Pfalz-Simmern, d. d. Neuburg, 12, VII. 1544 bei Salzer S. 78 Aum. 1: „Bitten wir in dieser hiichsten und letzten not etc. ${ }^{*}$

2) Über dieseu Landtag vergl. Lenz: Bucerbriefwechsel Bd. II, $\mathrm{S} .394 \mathrm{f}$. Linter den dort namentlich bezeichneten kurpfälzischen $\mathrm{Ab}$ gesandten befindet sich nicht der bei Druffel (S. 495) erwähnte Konz von Rechberg. Er war seit 1541 Hofmeister der Kurpfalz: rergl. J. G. Widder: Geogr. histor. Beschreibung der kurfürstl. Pfalz an Rhein (Frankfurt u. Leipzig 1786) Bd. 1, S. 44. Seine Stellung zur Beformation scheint sich mit dem Regierungswechsel in Heidelberg geändert zu haben: 1543 wird er uoch als Gegner der Protestanten bezeichnet: Lenz a. a. 1., Bd. II, S. 139, im Mai 1545 zählte ihn Bucer unter die Anhänger der Reform: ebenda S. 348. Höchst wahrscheinlich rïhrt von dieser plötzlichen Gesinnnngsänderung Ottheinrichs nicht ganz unterdrïcktes Mißtrauen gegen Rechberg her: Draffel S. 499. Von 1522-1526 war ein Konrad ron Rechberg Hofmeister in Pfalz Neuburg; ob er mit dem späteren karpfälzischen Hofmeister identisch ist, weiB ich nicht. Salzer S. $39 \mathrm{f}$.

3) Lenz: Bd. II S. 395 und bes. Salzer S. 79.

4) Lenz: Bd. III S. 362 a. S. 378. 
daran. Bayern in den schmalkaldischen Bund aufzunehmen, während ein Jahr fruher nach dem stande der Verhandlungen zwischen Herzog Wilhelm und Landgraf Philipp und besonders nach dem Verhïltnis der Munchener Regierung zum kaiserlichen Kabinet fuir Fernerstehende eine solche Möglichkeit nicht ausgeschlossen sein nochte. ${ }^{1}$ ) Mubten doch die bayrischen Herzoge den Zorn Karls fürchten. da sie ihm kurz zuvor unter ganz nichtigem Vorwande in seinem franzïsischen Feldzuge jegliche Lnterstuitzung versagt hatten.

II.

In mehrfacher Hinsieht wird das Aktenstück durch diese fribhere Datierung für dit Gesohichte Ottheinrichs und auch indirekt für die späteren Ereignisse in der Kurpfalz bedeutsam. Über seine Entstehung sei nur soviel gesagt. daB es ron der Neuburger Regierung ausgeht und zwar in Abwesenheit Ottheinrichs. wenn auch wohl nicht ohne seine Mitwirkung aufgesetzt worden ist.

Deutlich erkennen wir aus dem Memorial, daß das Mißtrauen Ottheinrichs gegen seinen Oheim, Kurfürst Friedrich, das später in gegenseitigen $\mathrm{HaB}^{2}$ ) ausgeartet zu sein scheint, schon zur Zeit seiner Übersiedelung nach Heidelberg bestand, also nicht erst durch eine schlechte Behandlung während dieses ziemlich unfreiwilligen Aufenthaltes bervorgerufen worden ist. Denn aus welchem anderen Grunde sollte man sich Ottheinrichs Verlangen erklären, daB die Rïte seines Oheims ihm jetzt sehon mit rerpflichtet wturden, als aus der Furcht, abermals von seinem Verwandten um seine wohlbegrundeten Anrechte auf die Kur gebracht zu werden? An Bestrebungen dieser Art hat es bekanntlich nicht gefehlt. nicht nur nicht als man den von des Kaisers schwerer lingnade Betroffenen dahin draingen wollte, gegen

1) Lenz: Bd. III S. $344 \mathrm{f}$.

$\left.{ }^{2}\right)$ Vergl. Zeitschr. für die Geschichte des Oberrheins Bd. XXV, S. 269: „Friedrich habe (1555) gesagt: „hertzog Otthainrich sitzt doben [in Neuburg] und bitt gott alle tag, das ich sterben soll, und da es hertzog Christoff [ron Würtemberg] verantworten wellen, hab er gesagt: sweigt, ich hab kain großern feindt auf erden." 
eine Geldentschädigung seinen Ansprüchen zu entsagen. sondern auch vor and während des schmalkaldischen Krieges. als ganz geheime Verbandlungen unter den pfälzischen Agnaten dahin zielten. Ottheinrichs Anrechte auf das Seuburger Land einem anderen Mitgliede der Familie zu ubertragen. ${ }^{1}$ )

Sodam erfahren wir aus unserm Aktenstidek. daB die im August 1544 nach der Übergabe des Landes an dic Stände eingesetzte Neuburger Regierung von Anfang all hinter ihrem fruheren Herrn gestanden hat. wenn er die reformatorischen Bestrebungen in seiner neaen Heimat be. gunstigte. ${ }^{2}$ ) ja dab rielleicht sie es gewesen ist. welche ihn am meisten dazu gedrängt hat. die Annäherung der kurpfälzischen Politik an den schmalkaldischen Bund anzubahnen. Denn nicht anders kann man die Worte in unserem Memorial (S. j00) auffassen: ..Na gedacht ze sein. wie hingegen auch ain ruck und ain solche hinderhuet gesucht, dadurch den bairischen ir nachteiligs rorhaben underkomen und abgestrickt werden mïcht." da ein Bund mit

1) Vergl. die Bemerkung zn einer früheren Werbung Hans Landschads, Vogtes zu Mosbach. und Adam Culmans, kurpfälzischen Rechenschreibers, bei Pfalzgraf Wolfgang ron Zweibriicken: „No. Nachdem man sich, wie hiervorn gemeldet wurt; eines tags rerglichen uf Jitwoch nach trinitatis [23. VI] zu Heidelberg eintzukomen, und rolgende tag handlung furzumemen, di entlich dahiu gemeint gewesen, wie mein gnediger fïrst und her [Pfalzgraf Wolfgang] zu dem Fürstenthumb Neuburg kommen möchte, so hat doch solcher tag sein fürgang nit erlangt ${ }^{\text {" }}$ wegen Ausbruches des schmalkaldischen Krieges. Itünchener Reichs-Archiv. Pfalz Neuburg. A. I So. 14 fol. 27.

C̈ber spätere Versuche (Dezember 1546), dem Bruder Friedrichs, Pfalzgraf Wolfgang, Statthalter in der Oberpfalz, das Neuburger Land zuzuxeisen vergl. Druffel: des Viglius rau Zwichem Tagebuch (München 1877) S. 225, Anm. 29.

2) So hören wir, daß bei Einführung der Reformation in der Kurpfalz im Frïhjahr 1546 die im Neuburger Lande zu grunde gelegte Nürnberger Kirchenordnung auch hier zur Anwendung gelangte, ohne Zweifel doch auf die Anregung Ottheinrichs hin. Vergl. Friedensburg: Nuntiaturberichte I, Bd. IX S. 32 Anm. 3, sowie Hans Rott: Friedrich II ron der Pfalz und die Reformation (Heidelberg 1904) S. $60 \mathrm{f} .$, anch Aum. 130, sowie S. $132 \mathrm{ff}$. 
dem Kaiser zum Schutze des Evangeliums natiurlich ausgeschlossen war, und eine andere Vereinigung in Deutschland. an welche man Anlehnung hätte suchen können. nicht existierte. Der limschwung in der politischen Haltung der Nouburger Regierung muB ziemlich plötzlich erfolgt sein: anfangs hatten statthalter und Regenten abgelehnt, die Iruheren Verhandlungen Ottheinrichs uber seinen Eintritt in den schmalkaldischen Bund ihrerseits fortzufuhren. ${ }^{1}$ ) da die drohende Nachbarschaft Bayerns in Verbindung mit der drückenden. für das kleine $I$,and unverhältnismäBig großen schuldenlast jeglichen nach außen hin provozierenden Schritt roll selbst zu verbieten schien. Doch die feste C̈berzeugung. daß der barrischen Herzöge ${ }^{2}$ ) auf den Erwerb von Neuburg hinzielende Politik nach wie vor feindlich bleiben werde, wird den Neuburger Ständen bald klar gemacht haben, daß nur die Anlehnung an den schmalkaldischen Bund ihnen Rettung vor den bayrischen Machenschaften in Aussicht stelle. Wenn auch die Wahl Kurfurst Friedrichs zum Erbschutz- und Schirmherrn der Neuburger Landschaft ${ }^{3}$ ) erst zu einem etwas späteren Termine erfolgte. als die Datierung des vorliegenden Memorials anzusetzen ist, ins Auge gefaBt haben wird man sie schon fruher, denn schon aus unserer Lrkunde tritt uns ein Gefuhl enger $Z_{a-}$ sammengehörigkeit mit der Rheinpfaly entgegen. Es war ein klug berechneter Zug. dab Statthalter und Regenten an Friedrich die Aufforderung gelangen ließen, seinerseits auch den bayrischen Bestrebungen entgegenzutreten. Klugerweise schlugen sie in ihrer Mahnung die fur sein furstliches und dynastisches Ehrgefuhl empfindlichste Saite an, wenn sie die Absichten der Munchener Wittelsbacher auf

1) Kiezler: Geschichte Baierns Bd. IV, S. 328.

*) Auch daB in dem Memorial immer noch von ${ }_{n}$ den $^{\mu}$ bayrischen Herren die Rede ist, spricht für die frühere Datierang. Herzog Ludwig starb bekanntlich an 21. April 1545.

3) Salzer a. a. O. S. 79. Die Wahl erfolgte am 30. Januar 1545. DaB Friedrich schon ror seiner Wabl zum Erbschutzherrn bein kaiserlichen Hof zn Gunsten des Neuburger Landes tätig war, berichtet r. Druffel: Karl V. and die römische Kurie. 2. Abt. S. 4 (Abhandlnngen d. kgl. bayr. Ak. d. W. III. Klasse, Bd. XVI. Abt. 1). 
das benachbarte Neuburger Land und der Herzögc Feindschaft gegen ihren sichwager Ottheinrich. die loch ihren Crsprung in erster linie in dessen Religionswechsel hatte. in rngsten Zusammenhang mit den ehrgeizigen Bestrehungen auf die pfälzische Kurwiurde brachten. Die Politik. welcher Ottheinrich in den Jahren seiner Verbannung am Heidelberger Hofe das Wort geredet hat, und welche eine kurze Zoit lang die kurpfälzische Diplomatie unmittelbar vor Ausbruch des schmalkaldischen Krieges verfolgt hat. finden wir in diesem Aktenstück bereits vorgezeichnet.

Auf einen nicht unwesentlichen Punkt sei zun Schluß noch hingewiesen. Die Vorschläge der Neuburger Regierung. durch Verhandlung beim Kaiser eine Garantierung der Kur für alle pfälzischen Agnaten durchzusetzen unter gleichzeitiger ,Mitbelehnung in Falle der Notwendigkeit für Ottheinrich. dessen Bruder Philipp und für Wolfgang Statthalter ${ }^{1}$ "* sind bis zu einem gewissen Grade enthalten in dem Heidelberger Vertrage vom 11. Februar 154;.") wonach Kurfürst Friedrich, Ottheinrich für sich und seinen Bruder Philipp. die Herzöge Johann ${ }^{3}$ ) ron Pfaly-Simmern und Wolfgang von Pfalz-Zweibrücken sich entschlossen zu dem einem Ziele vereinigten, die Kur gegenuber den Bestrebungen der Munchener Wittelsbacher ihren Familien zu erhalten.

Bei unserer fruheren Datierung der Urkunde erscheinen die Neuburger Regierung und mithin auch Ottheinrich als die intellektuellen L'rheber jene's Heidelberger Vertrages.

1) Muffat a. a. O. S. 293.

*) Der Heidelberger Tertrag findet sich abgedruckt bei Tolner: codex diplomaticus Palatinus (Frankfurt a. M. 1700) S. 166 f., nr. 222.

3) Es war wohl eine Erinnerung an die Hartnäckigkeit Johanns bei den Verhandlungeu über die Übergabe des Neuburger Landes [vergl. Salzer S. 78], weun Ottheinrich riet, mit ihm „in hochster geheim personlich" zu verhandeln. Druffel a. a. O. S. 500 . 